\title{
NO ENVEJECEMOS IGUAL: LA RELIGIOSIDAD Y EL GÉNERO EN ADULTOS MAYORES DEL NORESTE DE MÉXICO
}

\section{AGE DOES NOT EQUAL: RELIGION AND GENDER IN OLDER ADULTS OF THE NORTHEAST OF MEXICO}

\author{
Jesús Acevedo Alemán ${ }^{1}$ \\ jesusaceve@hotmail.com \\ José González Tovar ${ }^{2}$ \\ josegonzaleztovar@uadec.edu.mx
}

\begin{abstract}
Resumen
La religiosidad y el género representan, en la actualidad, dos grandes desafíos para los estudiosos del tema del envejecimiento; bajo esta perspectiva, el presente documento tiene como objetivo el exponer los resultados finales en relación con las condiciones de vida en las cuales se encuentran los adultos mayores de la ciudad de Saltillo, Coahuila, México, en dichos factores y cómo se encuentran relacionados. El estudio consideró una muestra de 1.500 habitantes mayores de 65 años. Según los resultados de la investigación, se concluye que el proceso de envejecimiento y las variables, las cuales forman parte de este proceso, son elementos que se ven influidos por el género de pertenencia de los Adultos Mayores (АM) por la religiosidad; por lo tanto, el rol tradicional que tienen en su familia afecta la manera en que se da el afrontamiento de la cotidianidad en el envejecimiento; y de ahí la importancia del contraste de estos resultados con los roles tanto individuales como con la familia en conjunto.

Palabras claves: envejecimiento, religiosidad, género y adulto mayor
\end{abstract}

\begin{abstract}
Religiosity and gender currently represent two major challenges for scholars of the issue of ageing, so this document is intended to expose the results in relation to the conditions of life in which the adults elderly of the city of Saltillo, Coahuila, Mexico are such factors and how are related. The study considered a sample of 1,500 people over the age of 65. According to the results of the investigation it was concluded that the aging process and the variables that are part of this process are elements that are influenced by the gender and religiosity, therefore their traditional role in your family affects the way is given the coping with everyday life in aging and hence the importance of the contrast of these results with the roles both individual with the family as a whole.
\end{abstract}

Key words: Aging, religion, gender and older adults

1 Facultad de Trabajo Social, Universidad Autónoma de Coahuila.

2 Escuela de Psicología Unidad Saltillo, Universidad Autónoma de Coahuila. 


\section{Introducción}

La problemática del adulto mayor en México está caracterizada - entre otros factores - por la intersección entre las problemáticas de la vejez, el género y la pobreza, que según Salgado y Wong (2007) se dan dentro de un contexto de escasa protección institucional y profundas desigualdades sociales en áreas urbanas y rurales. Los mismos autores destacan que las divergentes trayectorias de vida de los hombres y las mujeres los colocan en riesgos particulares y en situaciones diferentes de vulnerabilidad, por lo que las políticas y programas de salud deben adoptar una visión de problemas los cuales son comunes para ambos sexos, pero reconocer, al mismo tiempo, que sería necesario diseñar políticas separadas por género.

Márquez (2007) agrega que en México la representatividad poblacional del sector de los adultos mayores avanza a una velocidad vertiginosa y destaca que esta tendencia permite anticipar que, si en 2005 había 2 millones de adultos mayores, para el año 2050 uno de cada cuatro mexicanos será un anciano; al complementar que una parte importante de esta población de adultos mayores vive en situación de pobreza, dos de los cinco millones de hogares están en esta situación. Otra característica es que, según cifras del Instituto Nacional de Estadística y Geografía (INEGI) (2005) el 55\% de los adultos pobres corresponde a mujeres, lo cual es necesario tener siempre presente porque varones y mujeres no envejecen igual, su experiencia es diferente debido, entre otras razones, a los papeles genéricos que cada uno debe desempeñar en la sociedad.

En este sentido, es relevante identificar la experiencia del envejecimiento en las personas mayores, desde visiones diferenciadas por la condición social de género, en las cuales no sólo se perciba a la identidad masculina como fuerza de trabajo que provee a la familia y la femenina como la que suministra cuidados, atención y afecto, sino incluir visiones en las cuales se vean aspectos relevantes como la feminización del envejecimiento, la inequidad de género, la inseguridad económica, la discapacidad y los prejuicios de la sexualidad. Márquez (2007) agrega que independientemente de las visiones bajo las cuales se analice la problemática del género en adultos mayores, debe de estar presente la importancia de la familia y los roles de los integrantes en su conjunto, lo cual constituye el principal apoyo de las personas adultas mayores en zonas urbanas marginadas de México, lo cual permite además mejorar las necesidades de salud, su autocuidado y calidad de vida.

\section{La feminización del envejecimiento}

La vejez vista como un constructo social involucra la asignación de roles de acuerdo con la edad; por ejemplo, en cuanto a la participación en actividades económicas y sociales, según el género y las normas socioculturales. Salgado y Wong (2007) definen a la vejez como un proceso el cual involucra una serie de experiencias, muchas de ellas subjetivas, que se encuentran íntimamente relacionadas con las de vida, la edad biológica y la acumulación de riesgos a lo largo de los años vividos; asimismo, agregan que las mujeres conforman la mayoría entre los adultos mayores en casi todos los países del mundo (Sánchez, 2008). Este fenómeno es conocido como la 'feminización' del envejecimiento, el cual se observa universalmente porque las mujeres llegan a vivir más que los hombres. A pesar de que en el ámbito mundial nacen aproximadamente 105 niños por cada 100 niñas, estas los rebasan numéricamente alrededor de las edades de 30 a 40 años, y dicha ventaja numérica se hace mayor conforme avanza la edad. Datos de la Organización de las Naciones Unidas (ONU), citados por Salgado y Wong (2007), revelan que globalmente, alrededor del año 2000, había 190 mujeres por cada 100 hombres entre adultos mayores; por ejemplo, entre los octogenarios, el propio organismo estimó 181 mujeres por cada 100 hombres, aunque esta proporción aumenta de manera importante para los nonagenarios, con aproximadamente 287 mujeres por cada 100 hombres; y entre los adultos mayores de 100 años en los que se concentran nuevamente las mujeres: 386 mujeres por cada 100 hombres (INEGI, 2010).

Según Acevedo, Trujillo y López (2012), a la fecha, la población de adultos mayores, principalmente quienes viven en la pobreza, 
han recibido muy poca atención por parte de investigadores, prestadores de servicios y diseñadores de políticas públicas. Los autores exponen que existe un limitado número de estudios, los cuales se han enfocado en la relación entre envejecimiento, género y pobreza; sobre todo, la inequidad persistente a nivel mundial en el acceso a oportunidades para una mejor calidad de vida entre las mujeres ancianas quienes viven en situación de riesgo.

Esta situación destaca las inequidades del envejecimiento y el género, las cuales están íntimamente vinculadas con la pobreza a través de una interrelación muy compleja. Los propios autores enmarcan la complejidad de esta relación tridimensional (género, vejez y pobreza), la cual, según ellos, debe ser entendida como el resultado de una secuencia de acciones y experiencias sociales diferenciadas por sexo, que se inicia en edades tempranas y culminan en la vejez. De igual forma, finalizan al destacar que, en el mundo, las mujeres de más de 60 años tienden a vivir en pobreza más que los hombres, y el crecimiento de este grupo poblacional con grandes desventajas sociales continúa aumentando de manera importante, y da muestra de una inequidad de género en el proceso de envejecimiento (Salgado \& Wong, 2007; Montes de Oca, 2003).

\section{Inequidad de género}

El género en palabras de Acevedo, Trujillo y López (2012) se puede entender como una construcción social no natural que permea todos los rincones del tejido de una sociedad y considera todo un conjunto de características, oportunidades y expectativas que un grupo social asigna a las personas. Dicha construcción inicia con los respectivos roles de género, los cuales se le atribuyen al sujeto desde la infancia a través de la crianza y la educación escolar y familiar, fases que determinan las actitudes y conductas de hombres y mujeres en la sociedad (Montes de Oca, 2003).

De esta forma, el nivel de bienestar de las mujeres y los hombres mayores de 60 años es resultado de la trayectoria de vida que siguieron, así como del contexto social, económico e institucional que los rodeó (Márquez, 2007).
De acuerdo con esto, los hombres y las mujeres en su vejez se encuentran en diferentes estados de vulnerabilidad de acuerdo con el rol social, cultural y el nivel de protección institucional otorgado por la sociedad. Salgado y Wong (2007) expone que, históricamente, la falta de reconocimiento de las relaciones de género como característica fundamental de la jerarquía social ha actuado como una eficiente barrera para abordar adecuadamente los problemas de equidad que enfrentan los hombres y las mujeres, principalmente en la vejez (Gutiérrez, Serralde \& Guevara, 2007).

Montes de Oca (2003) expone que las mujeres mayores de 60 años tienden más a vivir sin una pareja que los hombres, esto se da, entre otras razones, como resultado de que, en promedio, las primeras vivan más años que los segundos (INEGI, 2005). De igual forma, agrega que estas tienden a formar uniones con varones de mayor edad que ellas, y tienden menos a formar nuevas uniones en casos de viudez, separación o divorcio (Palmore, 1991). Por esto, algunos adultos mayores se encuentran sin pareja, lo cual las coloca en una situación vulnerable, tanto desde el punto de vista económico como social. Además, el autor finaliza al replantear el hecho de que dicha vulnerabilidad por la falta de una pareja se observa sobre todo en países en desarrollo, donde la participación de las mujeres adultas mayores en la vida social y económica ha sido sumamente limitada, y su rol social depende, en gran medida, de su situación como hija, esposa o madre a lo largo de su vida (Nina, 2005). Lo anterior propicia en México, una gran división entre las funciones tradicionales de hombres y mujeres de los cohortes quienes están actualmente en edad avanzada, y sus consecuencias constituyen la problemática del género en la vejez y la religiosidad como soporte social (Acevedo, Trujillo \& López, 2012).

\section{Religiosidad como soporte social}

En México, $95.6 \%$ de la población se adscribe a alguna religión; se trata de una nación plurireligiosa donde conviven, a partir de la década de 1960, al menos 40 religiones establecidas, lo cual parece ser indicio de la importancia de la 
vida religiosa en la sociedad mexicana (INEGI, 2010). Según Rivera y Montero (2005), en principio, la religiosidad se vive en lo social como un cuerpo de conocimientos, comportamientos, ritos, normas y vínculos con lo divino (referente a la existencia de un Dios). Los autores agregan que la religiosidad posee un carácter directivo, al dotar al sujeto con los conocimientos necesarios fundamentales para ir en busca de lo divino (no necesariamente tras la experiencia de lo divino) a través del adoctrinamiento y la congregación con otros (Murillo \& Rapso, 2005).

De esta forma, la religiosidad es de naturaleza esencialmente social; hace las veces de contenedor de lo espiritual, de protector; es un soporte socio-cultural. En contraposición, señalan los autores, la naturaleza de la espiritualidad es singular, específica y personal; es incluso una dimensión que trasciende lo biológico, psicológico y social de la vida (Rivera \& Montero, 2005). La religiosidad, entonces, se concibe como un paso intermedio de socialización durante el cual el creyente se nutre del saber que dirigirá sus conductas en la búsqueda de la experiencia de lo divino, y da así paso a su espiritualidad (Acevedo, Trujillo \& López, 2012).

\section{La espiritualidad}

La espiritualidad es un constructo que se puede analizar por sus efectos en las respuestas de un sujeto con respecto a las condiciones de su existencia y en relación con lo divino. Rivera y Montero (2005) exponen que es un estado interno caracterizado por un sentimiento de integración con la vida y el mundo; es un proceso funcional dinámico que se desarrolla en la singularidad del sujeto y posteriormente se expresa en lo social, por lo cual se encuentra asociado directamente al bienestar físico y mental (Whetsell, Frederickcson, Aguilera \& Maya, 2005).

Lo anterior marca la sutil distinción entre la espiritualidad y la religiosidad; mientras la espiritualidad se asume como la experiencia de lo divino, la religiosidad es un paso intermedio de socialización durante el cual el creyente se nutre del saber que dirigirá sus conductas en la búsqueda de la experiencia de lo divino (Acevedo, Trujillo \& López, 2012). Cabe mencionar que la espiritualidad es un fenómeno complejo, se trata de una experiencia eminentemente personal, la cual en ocasiones no se puede explicar con palabras (fenómeno inefable). Si lo religioso implica un deber ser, lo espiritual implica simplemente ser, y en la actualidad parece existir una desvinculación entre ambos (Pargament, Smith, Koenig \& Pérez, 1998).

En México, Rivera y Montero (2007) han señalado que, en el catolicismo popular, la conducta personal de los sujetos está disociada; por un lado, por sus prácticas cotidianas regidas por sus códigos de ética y moral; y por el otro lado, por sus creencias religiosas y prácticas de culto; fidelidad al ritual que se sustituye en ocasiones, por los comportamientos sociales en la práctica cotidiana según le convenga al sujeto; conveniencia que puede llegar a ser ampliamente cuestionable, pero refleja la complejidad de los motivadores de las conductas de los sujetos, la cual, dicho sea de paso, le permite el cumplimiento de sus metas y proyectos de vida (Maestre, 2001).

Rivera y Montero (2005) agregan que en la sociedad mexicana se vive, como en casi todas las del mundo, una profunda crisis de valores, la cual se manifiesta en el retorno de lo religioso e implica más la búsqueda de un refugio espiritual que la verdadera creencia sobre la divinidad. De igual forma, agregan que el adulto mayor mexicano de principios del tercer milenio podría estar influenciado en una posición espiritual a partir de la cual lo religioso es eso, religiosidad, pero no espiritualidad. En otras palabras, se preocupa más por encontrar un refugio en los principios dogmaticos teológicos y templos religiosos, que una búsqueda verdadera de la elevación espiritual, acompañada de mayor reflexión sobre la existencia de los planos metafísicos (Barragán, Mejía \& Gutiérrez, 2007). En este sentido, Whetsell, Frederickcson, Aguilera y Maya (2005) destacan que la investigación sobre saneamiento holístico -derivado de la suma de todos los componentes y actores sociales- ha hecho énfasis en la importancia de establecer los aspectos espirituales como esenciales para la curación de enfermedades y el manejo de la salud.

Por su parte, los profesionales médicos han tratado de comprender el concepto de bienestar espiritual y su relación con la salud para la 
adaptación a la enfermedad y al proceso de envejecimiento. Enfermería, por ejemplo, como una disciplina holística, ha entendido la importancia de la espiritualidad en el proceso de curación. Los autores antes mencionados analizaron las definiciones de bienestar espiritual en la literatura de enfermería, las cuales parten de considerar la espiritualidad como un principio vivificante en la vida, y hacen énfasis en una relación trascendental con Dios u otros seres inmateriales. Así, concluyen que la falta de una definición unificada sobre espiritualidad - para enfermería- obstaculiza la investigación y el desarrollo de intervenciones espirituales por parte de los(as) enfermeros(as), lo cual puede generar el desarrollar intervenciones bajo planteamientos de vida espiritual saludable.

\section{Vida espiritual}

La vida espiritual parece jugar un importante papel en el afrontamiento del estrés y el mantenimiento de la salud del adulto mayor. Rivera y Montero (2007) han documentado que cuando la espiritualidad forma parte de la personalidad, tiende a expresarse en las estrategias de afrontamiento religioso con un impacto positivo en la salud, al ser éstas utilizadas eficientemente contra el estrés. Asimismo, destacan dos aproximaciones generales al estudio del afrontamiento religioso; la primera se encuentra presente en las formas específicas de afrontamiento (como el perdón, la purificación y la confesión, etc.); y la segunda, en los patrones de afrontamiento, como el afrontamiento religioso positivo y el religioso negativo; el primero asociado a mejores parámetros de salud, y el segundo a inadecuados parámetros (Tapia, Cravioto, Borges \& De la Rosa, 1996).

Los autores desarrollaron una investigación que evaluó la validez y confiabilidad de la escala de afrontamiento religioso (Brief-RCOPE) propuesta por Pargament, Smith, Koenig y Pérez (1998), en dos muestras de adultos mayores residentes de la Ciudad de México elegidas por disponibilidad en dos clínicas de salud. Dicho trabajo exploró la existencia de diferencias significativas en variables en función del sexo, edad, nivel educativo, religión, situación marital y laboral. La investigación incluyó información socio-demográfica, la escala de patrones positivos y negativos de métodos de afrontamiento religioso, y Brief-RCOPE con dos sub-escalas (afrontamiento religioso positivo y afrontamiento religioso negativo). Finalmente, los escritores hallaron diferencias significativas entre católicos y cristianos tradicionales las cuales parecen apuntar a que estos últimos tienden a desarrollar una relación con Dios más frecuentemente que los primeros, y recurren más a su fe para afrontar la soledad (Acevedo, Trujillo \& López, 2012).

Los resultados obtenidos aportan evidencia empírica sobre la manera en que viven los adultos mayores mexicanos, su vida espiritual (Alvarado, Templer, Bresler \& Thomas, 1995). Rivera y Montero (2007) enfatizan la importancia de continuar investigando en el área de la vida espiritual de las personas a fin de aprovechar eficientemente este importante recurso dentro de la psicoterapia y las estrategias de intervención preventiva con adultos mayores ante problemas como la soledad experimentada, muy especialmente por las mujeres solas y de bajo nivel educativo. Además, reconocen que, en este sector etario, el efecto positivo que la vida espiritual puede ejercer sobre ciertas entidades clínicas; tales como, depresión, desesperanza, ansiedad ante el envejecimiento, ansiedad ante la muerte y sobre la salud física en general presenta más fortalezas que debilidades.

Selye (1975) hace énfasis en que no son los estímulos estresantes en sí mismos, sino la reacción a estos lo que tiene un efecto negativo en nuestros cuerpos. En este sentido, Acevedo, Trujillo y López (2012) exponen que en las últimas cuatro décadas, al tratar de descubrir la manera cómo la mente puede ayudar a una persona a comprender el origen de su estrés y así reducirlo, los investigadores han buscado en las fuentes de la medicina tradicional que han sido utilizadas en la curación. Agregan que dichas técnicas, como la meditación y el yoga, entre otras, han ayudado a tranquilizar el espíritu y la mente, han permitido el aumentado el bienestar espiritual e interactúan con el cuerpo para su curación. Basados en las premisas anteriores, estos investigadores creen que el bienestar espiritual podría representar un papel muy importante en la promoción 
de la salud del individuo (Gala, Lupiani, Raja, Guillen, González, Villaverde \& Sánchez, 2002).

En América Latina, considerando el número de adultos mayores, es importante tener en cuenta la forma de dar apoyo y cuidado adecuado a estos hombres y mujeres, para asegurar una mayor calidad y dignidad en sus vidas. Con esta idea en mente, Whetsell, Frederickson, Aguilera y Maya (2005) señalan la importancia de tener presente los conceptos de bienestar espiritual y fortaleza, los cuales están relacionadas con la salud y que han sido investigadas como factores especialmente favorables para un envejecimiento exitoso. De igual forma, complementan al indicar que dicha conexión está asociada con la creencia de que hay una relación entre el cuerpo y la mente, la cual no es nueva, pero ha recibido mayor atención a medida que los investigadores en salud estudian los síntomas psicosomáticos, las uniones entre la mente y el sistema inmune; esta medicina mente-cuerpo permite considerar la interconexión posible de bienestar espiritual y fortaleza relacionada con la salud y cómo éstas contribuyen a la salud de los mayores y la calidad de sus vidas (Acevedo, Trujillo \& López, 2012).

Finalmente, los mismos autores expresan que convertirse en anciano puede significar muchas cosas para cada individuo, pues contiene varios factores: la carga genética, el ambiente en que se vive, los factores físicos, sociales, económicos y emocionales, entre otros. Sin embargo, volverse anciano es incuestionablemente un proceso de cambio constante e irreversible, la salud física, la pérdida de los seres queridos, el retiro forzoso, la situación económica y el grado de independencia en todas las áreas son elementos fundamentales para tener en cuenta cuando se mide el bienestar de las personas de edad avanza$\mathrm{da}$, las fortalezas con las que cuenta y la manera en cual mitiga su dolor (Whetsell, Frederickson, Aguilera \& Maya 2005).

Los antecedentes expuestos brindan elementos empíricos y justifican la problemática abordada en la investigación con respecto a los adultos mayores. Este proyecto tuvo como objetivos conocer las condiciones de vida y establecer las diferencias significativas en las actividades de los adultos mayores según el género y la participación en actividades relacionadas con profesar una religión.

\section{Método}

La presente investigación se situó en las líneas de estudios sobre la problemática de los grupos vulnerables, aplicados a la cuestión de los adultos mayores y su condición de vida. Para dar congruencia con el objetivo del estudio y para efectos metodológicos el estudio, se basó en la tradición de estudios cuantitativos con alcances comparativos. La estrategia de recolección de datos fue transversal expos-facto.

La muestra estuvo compuesta por 1,500 Adultos Mayores (AM), las edades oscilaron entre los 60 y 99 años. La edad media de la muestra fue de 67 años, con una desviación estándar de 5 años. Del total de la muestra, el $53.7 \%(\mathrm{n}=805)$ son mujeres y el $46.1 \%(n=692)$ son hombres; además, se reportaron 3 casos perdidos a este indicador. Hay diferencias significativas en la proporción de hombres y mujeres $(\chi=8,530, \mathrm{gl}=1$, $\mathrm{p}=0.003$ ). En cuanto a estructura familiar, el $0.8 \%$ reportó no tener hijos, $9 \%$ tiene uno o dos hijos, $33.9 \%, 31.3 \%$ indicó tener de cinco a seis hijos y el $24 \%$ tiene siete o más hijos. El $10 \%$ de los AM participantes vive solo, $43 \%$ lo hace con dos personas en su casa, $18.5 \%$ con tres personas, $11.3 \%$ con cuatro, $7.3 \%$ con cinco personas y sólo el $8.5 \%$ vive con más de seis personas en su hogar.

Para la recolección de los datos, se adaptó un instrumento de medición elaborado por Ribeiro (2008; Tamez \& Ribeiro, 2009), el cual está diseñado para diagnosticar la condición de vida de los adultos mayores, conformado por 213 reactivos distribuidos en 11 apartados, y se consideraron las variables de: 1) Datos Generales; 2) Composición e Historia Familiar; 3) Ayuda Recibida y Otorgada; 4) Vivienda; 5) Trabajo, Pensiones e Ingreso; 6) Seguridad Social y Servicios de Salud; 7) Salud; 8) Afectividad y Estado de Ánimo; 9) Actividades; 10) Maltrato y Negligencia; y 11) Comentarios finales (Acevedo, Trujillo \& López, 2012). Se debe aclarar que no todos los apartados del instrumento fueron procesados para este reporte. Luego, se tomaron en cuenta las variables de ayuda recibida y otorgada, 
afectividad y estado de ánimo así como actividades. Finalmente, los respectivos análisis estadísticos se procesaron en el programa estadístico SPSS versión 20; para el análisis se utilizaron pruebas de distribución y pruebas no paramétricas para dos y múltiples muestras independientes.

\section{Resultados}

A partir de los datos recabados con el instrumento descrito, se procedió al análisis de la prueba de distribución de Kolmogorov-Smirnov, ${ }^{3}$ que determina si existen diferencias significativas entre la forma de la distribución de los datos y la distribución normal teórica, esta prueba también ayudó a determinar el tipo de análisis estadístico adecuado para los datos. Se rechazó la hipótesis nula para los dos ejes analizados $\left(Z_{K-S} \geq 12,588\right.$, $p \leq 0.010$ ), por lo cual se decidió utilizar métodos

3 La prueba de Kolmogorov-Smirnov es una prueba no paramétrica para la igualdad de los continuos, pueden ser utilizados para comparar una muestra con una distribución de probabilidad de referencia (de una sola muestra de prueba K-S), o para comparar dos muestras (de dos muestras de prueba de K-S). La estadística de Kolmogorov-Smirnov cuantifica una distancia entre la función de distribución empírica de la muestra y la función de distribución acumulativa de la distribución de referencia, o entre las funciones de distribución empíricas de dos muestras. La prueba de KS de dos muestras es uno de los métodos no paramétricos más útiles y generales para la comparación de dos muestras, ya que es sensible a las diferencias tanto en ubicación y la forma de las funciones de distribución acumulativa empíricos de las dos muestras. paramétricos para el análisis de los resultados, además de que las escalas utilizadas son ordinales con tres opciones de respuesta. Se procesaron pruebas no paramétricas para el contraste por género y religión mediante las pruebas $\mathrm{U}$ de Mann-Whitney ${ }^{4}\left(\mathrm{U}_{M-W}\right), \mathrm{W}$ de Wilcoxon ${ }^{5}\left(\mathrm{~W}_{\mathrm{W}}\right)$ y $\mathrm{Z}$ de Kolmogorov-Smirnov $\left(Z_{K-S}\right)$, estas pruebas realizan comparaciones de variables para dos grupos (muestras independientes) cuando las variables tiene un nivel de medición ordinal.

\section{Estado de ánimo}

Según los resultados de las pruebas, hay diferencias significativas entre hombres y mujeres con respecto al estado de ánimo de los AM; así mismo, la muerte de un cónyuge y la enfermedad de algún pariente causan sufrimiento de manera diferente en los AM, hombres y mujeres (cuadro 1).

4 El Mann-Whitney U pruebas (también llamado el de Mann-Whitney-Wilcoxon, Wilcoxon la suma de rangos, o la prueba de Wilcoxon-Mann-Whitney). Representa una prueba estadística de tipo no paramétrico, prueba la hipótesis nula de dos poblaciones. Además permite comprobar la hipótesis nula, con una hipótesis alternativa, especialmente en una población particular.

5 La prueba de Wilcoxon de rangos, es una prueba de hipótesis de tipo no paramétrica, se utiliza cuando se comparan dos muestras relacionadas, o muestras emparejadas, o con mediciones repetidas de una sola muestra. Permite determinar si su media poblacional difieren. 
Cuadro 1

Análisis comparativo por genero del eje afectividad y estado de animo de los adultos mayores

\begin{tabular}{lcccccc}
\hline Indicador & U M-W & WW & P & ZK-S & p & U M-W \\
\hline $\begin{array}{l}\text { Últimamente (durante el último año) } \\
\text { ¿se considera usted feliz? }\end{array}$ & 258701.000 & 583116.000 & $-3,425$ & 0.001 & 1,389 & 0.042 \\
$\begin{array}{l}\text { ¿Le ha causado sufrimiento } \\
\text { la muerte del cónyuge o }\end{array}$ & 248007.000 & 572422.000 & $-3,971$ & 0.000 & 2,186 & 0.000 \\
alguna enfermedad de un pariente? & 257091.500 & 581506.500 & $-2,754$ & 0.006 & 1,567 & 0.015 \\
\hline
\end{tabular}

Fuente: Elaboración propia.

\section{Ayuda recibida y otorgada}

En el análisis comparativo de la ayuda recibida por género de los $\mathrm{AM}$, se encontraron diferencias significativas en la ayuda económica que se recibe, la percepción de suficiencia y el condicionamiento de ésta; asimismo diferencias en la ayuda recibida para hacer quehaceres domésticos, en especie como despensa, medicina, ropa y pagos varios; o instrumental para atención médica como sacar cita e ir a consulta (Cuadro 2).

Cuadro 2

Análisis comparativo por género del eje ayuda recibida y otorgada hacia los adultos mayores

\begin{tabular}{llcccc}
\hline \multirow{2}{*}{ Categoría } & \multicolumn{3}{c}{ Estadísticos de contraste } \\
\cline { 2 - 5 } & $\mathrm{U}$ M-W & WW & $\mathrm{p}$ & ZK-S & $\mathrm{p}$ \\
\hline Ayuda Económica & & & & & \\
\hline Recibe ayuda & 226300,000 & 550715,000 & 0.000 & 3,699 & 0.000 \\
\hline La ayuda recibida es suficiente & 229873,000 & 554288,000 & 0.000 & 3,188 & 0.000 \\
\hline La ayuda está condicionada a que usted da o haga algo a cambio & 250680,500 & 575095,500 & 0.000 & 1,990 & 0.001 \\
\hline Ayuda a quehaceres domésticos & & & & \\
\hline Recibe ayuda & 237625,500 & 562040,500 & 0.000 & 2,735 & 0.000 \\
\hline La ayuda recibida es suficiente & 228867,000 & 553282,000 & 0.000 & 3,287 & 0.000 \\
\hline Ayuda en especie & & & & \\
\hline Recibe ayuda para despensa, medicinas, ropa, pagos varios & 260104,000 & 584519,000 & 0.018 & 2,322 & 0.000 \\
\hline La ayuda recibida es suficiente & 241018,000 & 565433,000 & 0.000 & 2,602 & 0.000 \\
\hline La ayuda está condicionada a que usted dé o haga algo a cambio & 247077,500 & 571492,500 & 0.000 & 2,115 & 0.000 \\
\hline $\begin{array}{l}\text { Ayuda instrumental } \\
\text { La ayuda recibida para atención médica, sacar cita, que le }\end{array}$ & & & & \\
\hline lleve, le acompañe, que le apoye es suficiente & 244103,500 & 568518,500 & 0.000 & 2,329 & 0.000 \\
\hline $\begin{array}{l}\text { La ayuda está condicionada a que usted dé o haga algo a } \\
\text { cambio }\end{array}$ & 246540,000 & 570955,000 & 0.000 & 2,173 & 0.000 \\
\hline
\end{tabular}

Fuente: Elaboración propia. 


\section{Religiosidad}

Dentro del instrumento, se les preguntó a los AM si profesan alguna religión, el 93,5\% de la muestra que sí la profesan, un 5,7\% que no y un $0,7 \%$ que no contestaron; sumado a esto, el $47,5 \%$ de los adultos mayores asiste a servicios religiosos regularmente, seguido de un $45 \%$ que lo hace pocas veces y un $6,8 \%$ que nunca asiste a dichos servicios.

Se realizó una comparación entre los adultos mayores quienes asisten regularmente a servicios religiosos y quienes asisten pocas veces con la prueba $\mathrm{H}$ de Kruskall-Wallis, ${ }^{6}$ este análisis

6 La prueba de Kruskal-Wallis, es un método no paramétrico para probar si las muestras provienen de la misma distribución. Se utiliza para la comparación de más de dos muestras que son independientes, o no relacionadas. El equivalente paramétrica de la prueba de Kruskal-Wallis es el análisis unidireccional de la varianza (ANOVA). consiste en someter a prueba la hipótesis nula de diferencia entre las medianas de las dos muestras. Las variables comparadas fueron las correspondientes al eje actividades del AM (cuadro 3).

Al realizar la comparación, resultó que hay diferencias significativas entre quienes asisten regularmente, y pocas veces, a un servicio religioso, en cuanto a la frecuencia en la cual se reúnen con grupos de amigos, la asistencia a eventos sociales, practicar algún pasatiempo y recibir visitas en casa. Asimismo, hay diferencias en la frecuencia en que el AM participa en actividades recreativas con sus hijos nietos, con su pareja o con algún grupo o institución, además de tener un amigo o confidente con quien platicar. De igual forma, también hay diferencias en la preferencia del adulto por quedarse en casa y la percepción de que sus actividades han disminuido desde que tenía 50 años.

Cuadro 3

Análisis comparativo por religiosidad del eje actividades del adulto mayor

\begin{tabular}{lccccccc}
\hline & H- de Kruskall Wallis & \multicolumn{3}{c}{ Prueba de la Mediana } \\
\cline { 2 - 7 } Actividades & $\chi 2$ & $\mathrm{gl}$ & $\mathrm{p}$ & $\mathrm{Med}$ & $\chi 2$ & $\mathrm{gl}$ & $\mathrm{p}$ \\
\hline Se reúne usted con un grupo de amigo & 91,628 & 2 & 0.000 & 2,00 & 61,260 & 2 & 0.000 \\
\hline Asiste a eventos sociales o actividades recreativas & 215,771 & 2 & 0.000 & 2,00 & 91,590 & 2 & 0.000 \\
\hline Practica algún tipo de pasatiempo & 143,584 & 2 & 0.000 & 2,00 & 56,274 & 2 & 0.000 \\
\hline Recibe vistas en la casa & 96,028 & 2 & 0.000 & 1,00 & 86,907 & 2 & 0.000 \\
\hline $\begin{array}{l}\text { Participa con alguno de sus hijos o nietos en alguna } \\
\text { actividad recreativa }\end{array}$ & 87,640 & 2 & 0.000 & 2,00 & 135,509 & 2 & 0.000 \\
\hline Realiza actividades recreativas o sociales con su pareja & 19,218 & 2 & 0.000 & 2,00 & 50,976 & 2 & 0.000 \\
\hline Prefiere quedarse en casa que salir & 7,900 & 2 & 0.019 & 2,00 & 20,027 & 2 & 0.000 \\
\hline Realiza quehaceres domésticos & 218,857 & 2 & 0.000 & 1,00 & 237,394 & 2 & 0.000 \\
\hline $\begin{array}{l}\text { Considera que sus actividades han disminuido desde que } \\
\text { tenía 50 años }\end{array}$ & 3,242 & 2 & 0.198 & 2,00 & 19,467 & 2 & 0.000 \\
\hline $\begin{array}{l}\text { Participa con algún grupo o institución en actividades } \\
\text { Tiene algún amigo(a) o confidente con quién pueda }\end{array}$ & 321,223 & 2 & 0.000 & 2,00 & 141,586 & 2 & 0.000 \\
\hline platicar de sus cosas personales & 97,064 & 2 & 0.000 & 1,00 & 98,309 & 2 & 0.000 \\
\hline
\end{tabular}

Fuente: Elaboración propia. 


\section{Ayuda necesitada, recibida y condicionada}

Finalmente, también hay diferencias a partir de la asistencia a servicios religiosos en la ayuda necesitada, recibida, suficiente y condicionada en aspectos económicos, para realizar quehaceres domésticos, funcional para caminar, ir al baño, asearse o bañarse, tomar medicamentos, para levantarse de la cama, alimentarse y ayuda en especie como comida, ropa y medicinas (cuadro 4).

Cuadro 4

Análisis comparativo por religiosidad para el eje ayuda recibida y otorgada al adulto mayor

\begin{tabular}{|c|c|c|c|c|c|c|c|}
\hline \multirow{2}{*}{ Categoría } & \multicolumn{3}{|c|}{ H- de Kruskall Wallis } & \multicolumn{4}{|c|}{ Prueba de la Mediana } \\
\hline & $\chi^{2}$ & $\mathrm{gl}$ & $\mathrm{p}$ & Med & $\chi^{2}$ & $\mathrm{gl}$ & $\mathrm{p}$ \\
\hline Necesita ayuda económica & 14,997 & 2 & 0.000 & 1,00 & 16,443 & 2 & 0.000 \\
\hline Recibe ayuda económica & 51,582 & 2 & 0.000 & 1,00 & 67,179 & 2 & 0.000 \\
\hline La ayuda económica recibida es suficiente & 59,967 & 2 & 0.000 & 2,00 & 32,445 & 2 & 0.000 \\
\hline $\begin{array}{l}\text { La ayuda económica está condicionada a que usted da o } \\
\text { haga algo a cambio }\end{array}$ & 8,872 & 2 & 0.012 & 2,00 & 9,848 & 2 & 0.007 \\
\hline Necesita ayuda para quehaceres domésticos & 41,53 & 2 & 0.000 & 2,00 & 14,035 & 2 & 0.000 \\
\hline Recibe ayuda para quehaceres domestico & 68,665 & 2 & 0.000 & 1,00 & 62,23 & 2 & 0.000 \\
\hline La ayuda recibida es suficiente para los quehaceres & 71,611 & 2 & 0.000 & 1,00 & 61,864 & 2 & 0.000 \\
\hline Necesita ayuda funcional para caminar & 16,435 & 2 & 0.000 & 2,00 & 13,194 & 2 & 0.001 \\
\hline Recibe ayuda para caminar & 17,083 & 2 & 0.000 & 2,00 & 15,348 & 2 & 0.000 \\
\hline Necesita ayuda para ir al baño & 18,454 & 2 & 0.000 & 2,00 & 15,221 & 2 & 0.000 \\
\hline Recibe ayuda para ir al baño & 18,038 & 2 & 0.000 & 2,00 & 16,101 & 2 & 0.000 \\
\hline Necesita ayuda funcional para asearse o bañarse & 20,291 & 2 & 0.000 & 2,00 & 15,048 & 2 & 0.000 \\
\hline Recibe ayuda para asearse o bañarse & 19,052 & 2 & 0.000 & 2,00 & 14,924 & 2 & 0.000 \\
\hline Necesita ayuda funcional para tomar medicamentos & 19,617 & 2 & 0.000 & 2,00 & 17,499 & 2 & 0.000 \\
\hline Recibe ayuda para tomar medicamentos & 15,643 & 2 & 0.000 & 2,00 & 15,468 & 2 & 0.000 \\
\hline Necesita ayuda para levantarse de la cama o acostarse & 18,748 & 2 & 0.000 & 2,00 & 14,698 & 2 & 0.001 \\
\hline Recibe ayuda para levantarse de la cama o acostarse & 20,064 & 2 & 0.000 & 2,00 & 15,448 & 2 & 0.000 \\
\hline Necesita ayuda para alimentarse & 22,377 & 2 & 0.000 & 2,00 & 17,472 & 2 & 0.000 \\
\hline Recibe ayuda para alimentarse & 14,767 & 2 & 0.000 & 2,00 & 15,462 & 2 & 0.000 \\
\hline Necesita ayuda en especie & 19,405 & 2 & 0.000 & 2,00 & 23,716 & 2 & 0.000 \\
\hline
\end{tabular}

Fuente: Elaboración propia. 


\section{Conclusiones}

Al seguir los hallazgos de Salgado y Wong (2007), se valida el planteamiento de que las mujeres conforman la mayoría de los AM en casi todos los países del mundo, pues según la conformación de la muestra, la mayor parte de esta fue de mujeres $(53,7 \%$ son mujeres y el $46,1 \%$ hombres).

Según los resultados de la investigación, se concluye que el proceso de envejecimiento y las variables que forman parte de este proceso son elementos que se ven influidos por el género de pertenencia de los AM y, por lo tanto, el rol tradicional que tienen en su familia afecta la manera en que se da el afrontamiento de la cotidianidad en el envejecimiento; de ahí la importancia del contraste de estos resultados con los roles tanto individuales como con la familia en conjunto (Márquez, 2007). Asimismo, las actividades realizadas por el adulto mayor en su cotidianidad manifiestan el nivel de bienestar, de placidez, complacencia y hasta cierto punto del cumplimiento de metas personales, las cuales son un reflejo empírico del nivel de felicidad que tiene (Yuste, Rubio \& Aleixandre, 2004).

Las diferencias también se presentaron en el tipo de ayuda que reciben los AM, pues existen diferencias por género en la ayuda recibida y otorgada en aspectos económicos, domésticos, instrumentales y en especie. Estudios realizados de forma longitudinal han destacado la relación del sexo del AM con respecto a los niveles de felicidad o de bienestar subjetivo y además lo han relacionado con fenómenos como el suicidio (Koivumaa-Honkanar, Koskenvuo \& Kaprio, 2003 citados por Moyano \& Ramos, 2007), situación que se valida en los aspectos evaluados en este estudio.

Se destaca además que en la vida del AM, la religiosidad ${ }^{7}$ es una parte importante de su quehacer cotidiano, esto se sostiene a partir de la frecuencia a la que acuden a los servicios religiosos. Actividad que se distingue, frente al resto que realiza, como pueden ser las enfocadas al ocio,

7 Variable relacionada fuertemente con la ayuda recibida por el AM en aspectos económicos, domésticos y funcionales como el aseo personal, tomar medicamentos y alimentarse. al entretenimiento, la convivencia familiar y el desarrollo personal. Tales hallazgos dan respaldo empírico a la noción de religiosidad y sus implicaciones en el comportamiento cotidiano del AM (Rivera \& Montero, 2005) y se convierte es un factor protector ante la ansiedad en esta etapa de la vida (Acevedo \& González, 2013).

En resumen, los presentes resultados sostienen los argumentos de Acevedo, Trujillo y López (2012) quienes exponen que convertirse en anciano puede significar muchas cosas para cada individuo, y depende de los factores presentes (la carga genética, el ambiente en que se vive, los factores físicos, sociales, económicos y emocionales, entre otros). En tal sentido, es prioritario el identificar con certidumbre los procesos de construcción de la vida útil, o lo que represente envejecer, y de qué manera la religiosidad como soporte social y la feminización del envejecimiento puede contribuir en dicho proceso. Se destacó, a partir de los presentes resultados, que estos dos componentes deben ser considerados dentro del diseño de las políticas públicas, asî como de los programas de atención a las necesidades del adulto mayor, o en cualquier otra estrategia considerada para atender el bienestar social de este segmento poblacional.

\section{Referencias bibliográficas}

Acevedo, J., González, J. (2012) La familia versus la depresión en el adulto mayor: entre la esperanza y la muerte. p 277-294. En L. Cano. Investigación en trabajo social: retos y perspectivas. Universidad Nacional Autónoma de México. México.

Acevedo, J., Trujillo, Ma. A. y López, M. L. (2012). Envejeciendo dignamente. Una mirada hacia las condiciones de vida del adulto mayor. Plaza y Valdés. México.

Alvarado, K., Templer, D., Bresler, Ch. \& Thomas, S. (1995). The relationship of religious variables to death depression and death anxiety. Journal Clinical Psychology, 51: 202-214.

Barragán, A. J., Mejía, S. \& Gutiérrez, L. M. (2007). Dolor en los adultos mayores de 50 años: prevalencia y factores asociados. 
Salud Pública de México, 49(Sup. 4): 488-484.

Gala, F., Lupiani, M., Raja, R., Guillen, C., González, J., Villaverde, M. \& Sánchez, A. (2002). Actitudes psicológicas ante la muerte y el duelo. Una revisión conceptual. Cuadernos de Medicina Forense. 30: 39-50.

Gutiérrez, J., Serralde, A. \& Guevara, M. (2007). Prevalencia de desnutrición del adulto mayor al ingreso hospitalario. Nutrición Hospitalaria. 22:702-709.

Instituto Nacional de Estadística y Geografía (2005) Anuario de Estadisticas por Entidad Federativa. INEGI. México.

Instituto Nacional de Estadística y Geografía (2010) Censo de Población y Vivienda, 2000. México. INEGI

Márquez, M. (2007). Desprotección social en adultos mayores viviendo en la pobreza urbana. Salud Pública de México. 49(Número especial): 346-348.

Maestre, J. (2001). El dolor en el adulto mayor. Colombia Médica. 32:184-188.

Moyano, E. \& Ramos, N. (2007) Bienestar subjetivo: midiendo satisfacción vital, felicidad y salud en población chilena de la Región de Maule. Revista Universum. 22(2): 177-193.

Montes de Oca, V. (2003). El envejecimiento demográfico en el debate mundial: reflexión académica y política. Papeles de población. 35: 79-104.

Murillo, A. \& Rapso, M. (2005). Sexualidad en las personas mayores. Familia y persona de edad mayor. Red de Estudios de la Vejez. Puerto Rico.

Nina, R. (2005). Sabor a mi... Pareja y vejez. Familia y persona de edad mayor. Red de Estudios de la Vejez. Puerto Rico.

Pargament, K., B., Smith, H. Koenig \& Perez, L. (1998). Patterns of Positive and Negative Religious Coping with Major Life Stressors. Study Religion. 37(4): 721-724.
Palmore. E. (1991). Ageism, negative and positive (Second edition). New York: Springler Publishing Company.

Ribeiro, M. \& Mancinas, S. E. (Editores) (2009). Textos y contextos del envejecimiento en México: Retos para la familia y el Estado. Plaza y Valdés. México.

Rivera, A. \& Montero, M. (2007). Medidas de afrontamiento religioso y espiritualidad en adultos mayores mexicanos. Salud Mental.30(1): 39-47.

Rivera, A. \& Montero, M (2005). Espiritualidad y religiosidad en adultos mayores mexicanos. Salud Mental. 28(6): 51-58.

Salgado, V. \& Wong, R. (2007) Género y pobreza: determinantes de la salud en la vejez. Salud Pública. 49(4): 515-521.

Sánchez, D. (2008). Adultos mayores en la planeación del espacio turístico rural en Tamaulipas. Papeles de Población. 55:59-94.

Selye, H. (1975) The stress of life. New York. McGrawHill.

Tamez, B.M. \& Ribeiro, M. (2009). La solidaridad familiar hacia los adultos mayores en Monterrey, Nuevo León. p 245-276. En M. Ribeiro \& S. E. Mancinas. Textos y contextos del envejecimiento en México: Retos para la familia y el Estado. Plaza y Valdés. México.

Tapia, R., Cravioto, P., Borges, A. \& De la Rosa, B. (1996). Consumo de drogas médicas en población de 60 a 65 años en México. Encuesta Nacional de Adicciones 1993. Salud Pública de México. 18: 458-456.

Whetsell, M. V., Frederickson, K., Aguilera, P. \& Maya, J.L. (2005). Niveles de bienestar espiritual y de fortaleza relacionados con la salud en adultos mayores. Aquichan, 5(1): 72-85.

Yuste, N., Rubio, R. \& Aleixandre, M. (2004) Introducción a la psicogerontologia. Pirámide. Madrid. 